\section{Autoimmune and inflammatory diseases following COVID-19}

\section{Caroline Galeotti and Jagadeesh Bayry}

Emerging reports show that severe acute respiratory syndrome coronavirus 2 (SARS-CoV-2) infection precedes the appearance of various autoimmune and autoinflammatory diseases, including paediatric inflammatory multisystemic syndrome (PIMS) or multisystem inflammatory syndrome in children (MIS-C), thus adding to the growing mystery of this virus and raising questions about the nature of its link with autoimmune and autoinflammatory sequelae.

Refers to Verdoni, L. et al. An outbreak of severe Kawasaki-like disease at the Italian epicentre of the SARS-CoV-2 epidemic: an observational cohort study. Lancet https://doi.org/10.1016/S0140-6736(20)31103-X (2020).
Several emerging reports show that coronavirus disease 2019 (COVID-19), a pandemic respiratory infectious disease caused by severe acute respiratory syndrome coronavirus 2 (SARS-CoV-2), could lead to autoimmune and autoinflammatory diseases, such as paediatric inflammatory multisystemic syndrome (PIMS; which includes Kawasaki-like disease, Kawasaki disease shock syndrome, toxic shock syndrome, myocarditis and macrophage activation syndrome) in children ${ }^{1-6}$. In general, compared with adults, children are least affected by COVID-19 and have mild clinical symptoms. However, as reported by Verdoni et al. ${ }^{1}$ and others ${ }^{2-6}$, a sudden spike in PIMS (or multisystem inflammatory syndrome in children (MIS-C), as it is also known) has left clinicians and the scientific community with several questions about how and why it follows SARS-CoV-2 infection in certain children.

Infectious diseases have long been considered as one of the triggers for autoimmune and autoinflammatory diseases, mainly via molecular mimicry. A number of infectious agents have been implicated as the main trigger for the development of Kawasaki disease ${ }^{7}$, an acute vasculitis of unknown pathogenesis that occurs mainly in children $<5$ years old, and SARS-CoV-2 is now added to the list.

The European Centre for Disease Prevention and Control reported a total of 224 cases of PIMS across Europe as of 12 May 2020 (REF. $\left.{ }^{5}\right)$. A team from Bergamo, Italy, described a 30-fold increase in the incidence of Kawasaki disease and reported ten patients with Kawasaki-like disease, eight of whom were positive for IgG or IgM against SARS-CoV-2 $\left(R^{2} F^{1}\right)$. In London, UK, eight children reportedly had hyperinflammatory shock with features similar to atypical Kawasaki disease, Kawasaki disease shock syndrome or toxic shock syndrome; all eight were positive for SARS-CoV-2 antibodies ${ }^{2}$.

In a report issued on 14 May 2020, the French National Public Health Agency documented 125 cases of atypical PIMS occurring between 1 March and 12 May 2020 (REF. ${ }^{5}$ ); 65 of the affected children have tested positive for SARS-CoV-2 to date. Detailed investigations of some of these cases, including a study of 17 patients in Paris ${ }^{3}$ and a retrospective study of 35 patients across twelve French and one Swiss medical centre ${ }^{4}$, revealed that in addition to gastrointestinal symptoms, skin rashes, cervical lymphadenopathy, cheilitis and high levels of inflammatory markers, myocardial involvement was common ${ }^{3,4}$. Many patients presented with an incomplete form of Kawasaki disease.

In the USA, the New York City Health Department on 4 May 2020 reported that 15 children aged $2-15$ years had presented with symptoms of MIS-C, including persistent fever and increased levels of inflammatory markers, and many also had rash, abdominal ren were positive for SARS-CoV-2 infection $^{6}$. By 12 May 2020, the number of cases of suspected MIS-C had risen to 102 (REF. ${ }^{6}$ ).

All these reports indicate that the symptoms of COVID-19-associated PIMS overlap with Kawasaki disease, but affected patients also present with symptoms not commonly associated with Kawasaki disease. Therefore, it is critical to make the fine distinction between classical Kawasaki disease and Kawasaki disease associated with COVID-19 (KD-COVID-19). Italian paediatricians noticed that, compared with classical Kawasaki disease, patients with KD-COVID-19 were older and had gastrointestinal and meningeal signs; they also had leukopenia with marked lymphopenia, thrombocytopenia, increased ferritin and markers of myocarditis ${ }^{1}$. Other reports also recorded a high incidence of myocarditis and cardiac involvement in KD-COVID-19 (REFS $\left.{ }^{2-4}\right)$. The increased incidence of myocarditis highlights that patients with KD-COVID-19 are more severely ill and are often hospitalized in intensive care. Future investigations should aim to dissect the underlying molecular mechanisms that lead to PIMS following exposure to SARS-CoV-2 (BOX 1).

The clinical history of patients with PIMS suggests that many had previously experienced mild symptoms of COVID-19 or had contact with COVID-19-positive family members. In the French study ${ }^{3}$, the median period between COVID-19 symptoms and onset of PIMS signs was 42 days (range 18-79 days). Accordingly, most of the patients with PIMS were positive for IgM or IgG antibodies to SARS-CoV-2 when the autoinflammatory symptoms appeared. The presence of IgG, which typically appears $\sim 2$ weeks after primary infection, suggests a delayed onset of PIMS following primary infection. However, nasopharyngeal and oropharyngeal swabs from several patients with PIMS were positive pain, vomiting or diarrhea; ten of the 15 child-

\section{Box $1 \mid$ Key questions}

Several important questions arise about the autoimmune and autoinflammatory responses following severe acute respiratory syndrome coronavirus 2 (SARS-CoV-2) infection.

-What are the molecular mechanisms that trigger autoimmune and/or autoinflammatory responses following coronavirus disease 2019 (COVID-19)?

- What are the genetic predisposition factors implicated in COVID-19-associated paediatric inflammatory multisystemic syndrome (PIMS) or multisystem inflammatory syndrome?

- Are autoimmune and/or autoinflammatory responses associated with a particular variant form of SARS-CoV-2?

- What is the nature of the SARS-CoV-2 pathogen-associated molecular patterns that trigger autoimmune and/or autoinflammatory responses?

- Are the mechanisms implicated in the induction of high concentrations of IL-6 in PIMS and adults with severe COVID-19 similar or distinct? 
for SARS-CoV-2 (REFS $\left.{ }^{1-6}\right)$, indicating that PIMS might even occur during the late phase of COVID-19.

In adults, the spectrum of complications following COVID-19 is broader than in children and includes autoimmune diseases but their incidence is too rare. Idiopathic thrombocytopenic purpura, Guillain-Barré syndrome and autoimmune haemolytic anaemia have been recorded in one, five and seven patients respectively, 4-13 days following onset of COVID-19 symptoms ${ }^{8-10}$. Thus, in contrast to PIMS, autoimmune symptoms in adult patients appeared during the early, active phase of COVID-19.

Although a causal link between SARSCoV-2 and the appearance of autoimmune and autoinflammatory diseases has not yet been firmly established, it is suggested by the temporal association with the current COVID-19 pandemic and the history of exposure of affected patients to SARS-CoV-2. We can envisage two possibilities. First, SARS-CoV-2 could act as a direct trigger of autoimmune and/or autoinflammatory conditions either by molecular mimicry or other, unknown mechanisms. A fine analysis of homology between various antigens of SARS-CoV-2 and self-antigens, by use of in silico approaches and validation in experimental models, should be considered in order to confirm this hypothesis. A second possibility is that inflammation and dysregulated immune responses following SARS-CoV-2 infection might prompt other environmental insults to cause the observed pathologies in predisposed individuals. As mentioned, several pathogens have been proposed as triggers for Kawasaki disease and other autoimmune diseases in the past ${ }^{7}$. In fact, although the French ${ }^{3}$ and Italian ${ }^{1}$ studies suggest that patients are negative for common viruses that trigger Kawasaki disease, data from other European countries reveal that some patients with PIMS are also positive for viral (rhinovirus, adenovirus, Epstein Barr virus or human metapneumovirus) or bacterial (Streptococci or Staphylococci) pathogens ${ }^{5}$ that were previously implicated as triggers of Kawasaki disease.

The reports from the UK and France suggest that PIMS is more frequent in patients of Afro-Caribbean descent ${ }^{2,3}$, and cases of KD-COVID-19 have not been reported in Asian countries (where the incidence of Kawasaki disease is typically the highest), which suggests genetic predisposition could be a factor in the development of KD-COVID-19. Various genetic factors have been identified that predispose children for Kawasaki disease $^{7}$. Investigation of predisposing factors, for example, in genome-wide association studies, will provide mechanistic insights on the incidence of PIMS. Another area that requires attention is whether the appearance of PIMS or autoimmune responses is associated with a particular variant of SARS-CoV-2 (BOX 1). As autoinflammatory signs are sequelae to SARS-CoV-2 infection and mostly appear several weeks post-infection, highly sensitive and specific serological tests are critical for establishing a causal link between COVID-19 and PIMS.

Current reports indicate that the spectra of autoimmune and autoinflammatory conditions in SARS-CoV-2-infected populations are mostly responsive to intravenous immunoglobulin (IVIG) therapy ${ }^{7}$. Early diagnosis of COVID-19-linked autoimmune and autoinflammatory diseases, and prompt initiation of therapy, is crucial for successful recovery and preventing end-organ damage and fatality. However, compared with classic Kawasaki disease, resistance to IVIG therapy was more common in KD-COVID-19 (REFS ${ }^{1,3}$ ) and needed adjunct steroids. To resolve the severe inflammatory state associated with PIMS, three children in the French-Swiss study ${ }^{4}$ were successfully treated with anakinra, an IL-1 receptor antagonist (D. Bonnet, personal communication). Patients with PIMS also have elevated IL-6 concentrations compared with normal controls ${ }^{1,3,4}$, similar to what is seen in severely ill adults with COVID-19. IL- 6 has been implicated in the pathogenesis of myocarditis. Importantly, IL-6-targeted immunotherapies produced favourable responses in adults severely ill with COVID-19. Therefore, inhibition of IL-6 signalling in PIMS by blocking IL-6 receptor by use of either tocilizumab (a humanized monoclonal antibody) or sarilumab (a fully human monoclonal antibody) could also be considered in patients with PIMS.

The current COVID-19 pandemic has given rise to many surprises, including the appearance of PIMS, with clinical and biochemical features distinct from that of classical Kawasaki disease and affecting children up to 17 years old. Although only a small proportion of SARS-CoV-2-infected children subsequently develop symptoms of PIMS (or MIS-C), most of them require intense clinical management because of the severity of the disease. As fundamental aspects of PIMS remain largely unknown, future investigations will require close interaction among various disciplines including paediatrics, internal medicine, rheumatology, immunology, genetics, infectiology, cardiology and epidemiology.

Caroline Galeotti ${ }^{1}$ and Jagadeesh Bayry (D) $^{2 \times}$

${ }^{\prime}$ Service de Rhumatologie Pédiatrique, Centre de Référence des Maladies Auto-Inflammatoires Rares et des Amyloses, CHU de Bicêtre, le Kremlin Bicêtre, France.

Institut National de la Santé et de la Recherche Médicale, Centre de Recherche des Cordeliers, Sorbonne Université, Université de Paris, Paris, France.

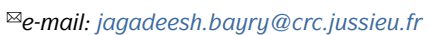
https://doi.org/10.1038/s41584-020-0448-7

1. Verdoni, L. et al. An outbreak of severe Kawasaki-like disease at the Italian epicentre of the SARS-CoV-2 epidemic: an observational cohort study. Lancet https://doi.org/10.1016/S0140-6736(20)31103-X (2020).

2. Riphagen, S., Gomez, X., Gonzalez-Martinez, C., Wilkinson, N. \& Theocharis, P. Hyperinflammatory shock in children during COVID-19 pandemic. Lancet 395, 1607-1608 (2020)

3. Toubiana, J. et al. Outbreak of Kawasaki disease in children during COVID-19 pandemic: a prospective observational study in Paris, France. medRxiv https://doi.org/10.1101/2020.05.10.20097394 (2020).

4. Belhadjer, Z. et al. Acute heart failure in multisystem inflammatory syndrome in children (MIS-C) in the context of global SARS-CoV-2 pandemic. Circulation https://doi.org/10.1161/CIRCULfrenchATIONAHA. 120.048360 (2020)

5. European Centre for Disease Prevention and Control. Paediatric inflammatory multisystem syndrome and SARS-CoV-2 infection in children. ECDC https://www. ecdc.europa.eu/sites/default/files/documents/covid19-risk-assessment-paediatric-inflammatorymultisystem-syndrome-15-May-2020.pdf (2020).

6. The Centers for Disease Control and Prevention (CDC) Health Alert Network. Multisystem Inflammatory Syndrome in Children (MIS-C) Associated with Coronavirus Disease 2019 (COVID-19). CDC https://emergency.cdc.gov/ han/2020/han00432.asp (2020).

7. Galeotti, C., Kaveri, S. V., Cimaz, R., Koné-Paut, I. \& Bayry, J. Predisposing factors, pathogenesis and therapeutic intervention of Kawasaki disease Drug Discov. Today 21, 1850-1857 (2016).

8. Zulfiqar, A. A., Lorenzo-Villalba, N., Hassler, P. \& Andres, E. Immune thrombocytopenic purpura in a patient with Covid-19. N. Engl. J Med. 382, e43 (2020).

9. Toscano, G. et al. Guillain-Barré syndrome associated with SARS-CoV-2. N. Engl. J. Med. https://doi.org/ 10.1056/NEJMc2009191 (2020).

10. Lazarian, G et al. Autoimmune haemolytic anaemia associated with COVID-19 infection. Br. J. Haematol. https://doi.org/10.1111/bjh. 16794 (2020).

\section{Acknowledgements}

J.B. acknowledges support from the COVID emergency fund from Université de Paris.

Competing interests

The authors declare no competing interests. 